\title{
Prevention and Management of Thrombosis in BCR/ ABL-Negative Myeloproliferative Neoplasms
}

\author{
Anna Falanga ${ }^{1,2}$ Marina Marchetti ${ }^{1}$ Francesca Schieppati ${ }^{1}$ \\ ${ }^{1}$ Department of Immunohematology and Transfusion Medicine, \\ Hospital Papa Giovanni XXIII, Bergamo, Italy \\ 2 University of Milano-Bicocca, Department of Medicine and Surgery, \\ Monza, Italy \\ Address for correspondence Anna Falanga, MD, Department of \\ Immunohematology and Transfusion Medicine, Hospital Papa \\ Giovanni XXIII, Piazza OMS 1, 24127 Bergamo, Italy \\ (e-mail: annafalanga@yahoo.com).
}

Hämostaseologie 2021;41:48-57.

\begin{abstract}
Keywords

- management of thrombosis

- prevention

- thrombosis

- myeloproliferative neoplasms

Myeloproliferative neoplasms (MPNs) are clonal disorders of the hematopoietic stem cell. Classical BCR/ABL-negative MPNs include polycythemia vera (PV), essential thrombocythemia (ET), and primary myelofibrosis (PMF). Thrombotic events are a major cause of morbidity and mortality in these patients. Pathogenesis of blood clotting activation involves various abnormalities of platelets, erythrocytes, and leukocytes, as well as dysfunctions of endothelial cells. Patients with MPN can be stratified in "high risk" or "low risk" of thrombosis according to established risk factors. ET and PV clinical management is highly dependent on the patient's thrombotic risk, and a risk-oriented management strategy to treat these diseases is strongly recommended. In this review, we give an overview of risk factors, pathogenesis, and thrombosis prevention and treatment in MPN.
\end{abstract}

\section{Introduction}

Myeloproliferative neoplasms (MPNs) are clonal disorders of the hematopoietic stem cell, characterized by an uncontrolled expansion of myeloid precursors in the bone marrow and an excess of differentiated erythrocytes, platelets, and leukocytes in the peripheral blood. According to the 2016 revised World Health Organization (WHO) Classification of myeloid neoplasm, classical BCR/ABL-negative MPNs include polycythemia vera (PV), essential thrombocythemia (ET), and primary myelofibrosis (PMF), with the novel subcategory "prefibrotic/ early-stage myelofibrosis" (pre-PMF). ${ }^{1}$ Besides the transformation into secondary myelofibrosis or acute myeloid leukemia (AML), thrombotic events are a major cause of morbidity and mortality in these patients. ${ }^{2}$

We will review the risk factors, pathogenesis, and management of thrombosis in BCR/ABL-negative MPNs.

\section{Thrombosis Incidence}

The rate of arterial and venous thrombosis in MPN patients has been estimated as 3-fold and 10-fold increased, respectively, compared with the general population. ${ }^{3}$ Arterial thromboses account for approximately two-thirds of total thrombotic events related to MPN, ${ }^{4}$ and include ischemic stroke, acute myocardial infarction, and peripheral arterial occlusions. The frequency of arterial events before or at initial presentation of MPN is approximately 16 to $27 \%$ in $\mathrm{PV},{ }^{5,6} 18 \%$ in ET, ${ }^{7}$ and $4 \%$ in $\mathrm{PMF}^{8}$ The cumulative rate of thrombotic events in the followup has been estimated of $5.5,1$ to 3 , and $1.75 \%$ patient-years in PV, ET, and PMF, respectively, ${ }^{6,8-10}$ and a cardiovascular (CV) mortality of $1.7 \%$ patient-years has been reported in PV. ${ }^{6}$ Venous thromboembolism (VTE) covers one-third of total thrombotic events in MPN, occurring in approximately $0.6 \%$ patient-years in ET and pre-PMF, ${ }^{11} 0.76 \%$ in $\mathrm{PMF}^{8}$ and $1 \%$ in PV. ${ }^{5}$ Events involving the venous system are deep vein thrombosis (DVT) of the lower extremities, pulmonary embolism (PE), splanchnic (hepatic, portal, and mesenteric), and cerebral vein thrombosis. ${ }^{12}$ In particular, the prevalence of splanchnic and cerebral vein thrombosis is unusually high among patients with MPN, ${ }^{13}$ and, as such, MPN diagnosis should always be considered if thrombosis manifests at an uncommon location. Thrombotic complications considerably affect MPN patients' received

November 16, 2020

accepted after revision

December 8, 2020 (c) 2021. Thieme. All rights reserved. Georg Thieme Verlag KG,

Rüdigerstraße 14,

70469 Stuttgart, Germany
DOI https://doi.org/

10.1055/a-1334-3259.

ISSN 0720-9355. 
prognosis, being an independent negative prognostic factor for survival in ET and PV. ${ }^{11,14}$ In PMF, the other major fatal and nonfatal competing events (i.e., acute leukemia transformation, infections, etc.) may obscure the negative prognostic effect of thromboembolism, and its real incidence. Nevertheless, an accurate risk assessment for thrombosis prevention represents a cornerstone of MPN management.

\section{Risk Factors for Thrombosis in Myeloproliferative Neoplasms}

\section{Clinical Risk Factors}

Older age and a history of thrombosis are well-established independent predictors of recurrent VTE in MPN. ${ }^{6,15}$ In the ECLAP study, the rate of CV complications in PV was significantly higher in patients aged more than 65 years or with a history of thrombosis than in younger subjects with no history of thrombosis. ${ }^{6}$ In ET patients, age $>60$ years and previous thrombosis were both independently associated with higher risk to develop major thrombosis in the long term. ${ }^{15}$ In MPN, the role of conventional CV risk factors for arterial thrombosis (i.e., obesity, hypertension, diabetes, dyslipidemia, and smoking) has been evaluated with conflicting results ${ }^{16}$; nevertheless, it is reasonable to assume that risk factors for atherosclerosis provide at least the same relative risk of the general population, and should be corrected in MPN patients.

\section{JAK2, CALR, and MPL Mutations}

$J A K 2$ is a cytoplasmic tyrosine kinase protein required for signal transduction. Two types of JAK2 mutations are associated with MPN. The gain-of-function V617F mutation in exon 14 is harbored by approximately 96,55 , and $65 \%$ of patients with PV, ET, and PMF, respectively, where mutations in exon 12 can be detected in 3\% of V617F-negative PV patients. ${ }^{17}$ The second most common mutation in MPN involves the calreticulin gene (CALR), and occurs in 25 to $35 \%$ of patients with PMF and 15 to $24 \%$ with ET. ${ }^{18}$ Finally, mutations in the TPO receptor gene MPL are present in $4 \%$ of ET and $8 \%$ of PMF cases. CALR and MPL mutations are rarely reported in PV. ${ }^{17}$ Several studies have evaluated the association between JAK2V617F and the severity of MPN disease. In particular, two meta-analyses have evidenced a correlation between JAK2 mutation and the risk of thrombosis in ET. ${ }^{19,20}$ Moreover, one study identified JAK2 mutation as an independent risk factor for arterial thrombosis. ${ }^{15} \mathrm{~A}$ reduced thrombotic risk in CALR-positive as compared with JAK2V617F-positive patients has been reported by several studies ${ }^{21-23}$; however, the inclusion of CALR mutational status into the international prognostic score for thrombotic risk assessment in ET (i.e., IPSET-thrombosis), which includes age more than 60 years, CV risk factors, and previous thrombosis, did not significantly modify the risk stratification of the original score. ${ }^{24}$ Thus, the absence of JAK2V617F and not necessarily the presence of CALR mutation or "triple-negative" mutational status (i.e., wild-type $J A K 2, C A L R$, and MPL) seem to be associated with lower risk of thrombosis compared with JAK2-mutated cases. ${ }^{25}$

\section{Elevated Blood Cell Counts}

Blood cell counts have been investigated as thrombophilic risk factors in MPN. Hyperviscosity, as a consequence of erythrocytosis, has been related to MPN prothrombotic state for a long time. ${ }^{26}$ Data supporting an association between elevated hematocrit and thrombotic events, however, have not been always concordant. ${ }^{27,28}$ Recently, in the CYTO-PV study, patients with a hematocrit less than $45 \%$ had a significantly lower rate of $\mathrm{CV}$ deaths and major thrombosis than those with a hematocrit of 45 to $50 \%{ }^{29}$ Moreover, a study from the Spanish Registry of Polycythemia Vera demonstrated that PV patients with higher phlebotomy requirements were at the highest risk of developing thrombotic events. ${ }^{30}$

While no study to date has demonstrated a statistically significant correlation between platelet count and thrombosis in either PV or ET, 6,7 extreme thrombocytosis (i.e., platelets $>1,000 \times 10^{9} / \mathrm{L}$ ) can favor hemorrhages in ET patients. ${ }^{31}$ This phenomenon has been attributed to the possible occurrence of acquired von Willebrand syndrome (AvWS), due to an increased clearance by platelets of the large von Willebrand factor multimers. ${ }^{32}$

A growing amount of evidence has been produced regarding the role of leukocytosis as a risk factor for thrombosis in MPN patients. ${ }^{9,33-36}$ Although a correlation between white blood cell (WBC) count at diagnosis and/or during the followup and the occurrence of arterial and venous events emerges in most of the studies on this topic, results are not homogeneous and easily comparable. A recent meta-analysis showed that the relative risk of thrombosis in the presence of leukocytosis is 1.59 , mainly accounting for ET and arterial thrombosis subgroups, with no significant effect on venous thrombosis alone. ${ }^{37}$ As the authors underlined, the lack of a clear cut-off value for WBC counts, the design of the studies never specifically aimed at assessing the role of leukocytosis, and the differences in sample size and duration of follow-up have led to inconclusive evidence. ${ }^{37}$ Thus, despite biological evidences, leukocytosis has never been formally included in risk models and prognostic scores.

\section{Risk Classification}

Based on the aforementioned studies, PV patients are classified as high risk if older than 60 years or present a history of thrombosis, and low risk in the absence of both risk factors. ${ }^{17}$ Until recently, the same risk stratification has been used in ET. ${ }^{38}$ Since the role of JAK2 mutation and CV risk factors have emerged as independent predictors of thrombosis in ET, ${ }^{15}$ these variables have been evaluated in risk stratification. First, JAK2 mutation along with CV risk factors has been proposed as part of the score in the IPSET-thrombosis classification. ${ }^{39}$ A subsequent analysis from the same group on a larger cohort of ET patients documented a weak contribution of CV risk factors in both low-risk and highrisk patients. ${ }^{40}$ Thus, current risk stratification in ET includes four categories: very low risk (age $\leq 60$ years, absence of previous thrombosis, JAK2 wild type); low risk (age $\leq 60$ years, absence of previous thrombosis, mutation of JAK2); intermediate risk (age $>60$ years, absence of previous 
thrombosis, JAK2 wild type), and high risk (age $>60$ years or previous thrombosis plus mutation of JAK2). ${ }^{17}$

\section{Mechanisms of Thrombosis in Myeloproliferative Neoplasms}

Even though vascular complications represent a major cause of morbidity and mortality, MPN-associated thrombophilia is poorly understood and currently believed to be multifactorial. Among the different mechanisms, the activated status of platelets, erythrocytes, and leukocytes arising from the clonal proliferation of hematopoietic progenitor cells, and the documented clotting activation, are considered major players in MPN-associated thrombosis. ${ }^{12}$ More recently, several reports indicate that a proinflammatory MPN milieu as well as excessive interactions between qualitatively abnormal vascular cells, including red blood cells (RBC), leukocytes, platelets, and endothelial cells (EC), are implicated in the generation of thrombotic events. ${ }^{41}$ The principal prothrombotic pathogenic mechanisms involved in MPN-associated thrombosis are described in -Fig. 1. As explained in the figure, several adhesion molecules are expressed by blood cells in MPN patients, mediating their reciprocal interaction and activation, and favoring thrombosis development. Recent studies have evaluated blocking antibodies to some of these molecules, like crizanlizumab targeting P-selectin, for other clinical conditions, ${ }^{42}$ and may provide a rationale for future studies exploring the use of such drugs to reduce thrombotic complications in MPN.

Of interest, JAK2V617F has been detected in mature EC from splenic and liver vein of PMF and PV patients with Budd-Chiari syndrome. ${ }^{43}$ Moreover, increased thrombus formation has been demonstrated in mouse models expressing JAK2V617F only in the endothelial compartment, overall suggesting that JAK2-mutant EC could contribute to the prothrombotic phenotype. ${ }^{41}$ Finally, elevated levels of circulating platelet-derived procoagulant microparticles (MPs), ${ }^{44}$ and the occurrence of an acquired activated protein C resistance, ${ }^{45}$ also contribute to hypercoagulability in these subjects.

\section{Prevention of Thrombotic Complications in Myeloproliferative Neoplasms}

Current treatment for PV and ET is aimed at preventing thrombotic complications, differently from PMF, where available therapies are directed to mitigate constitutional symptoms, splenomegaly, and anemia, or have curative

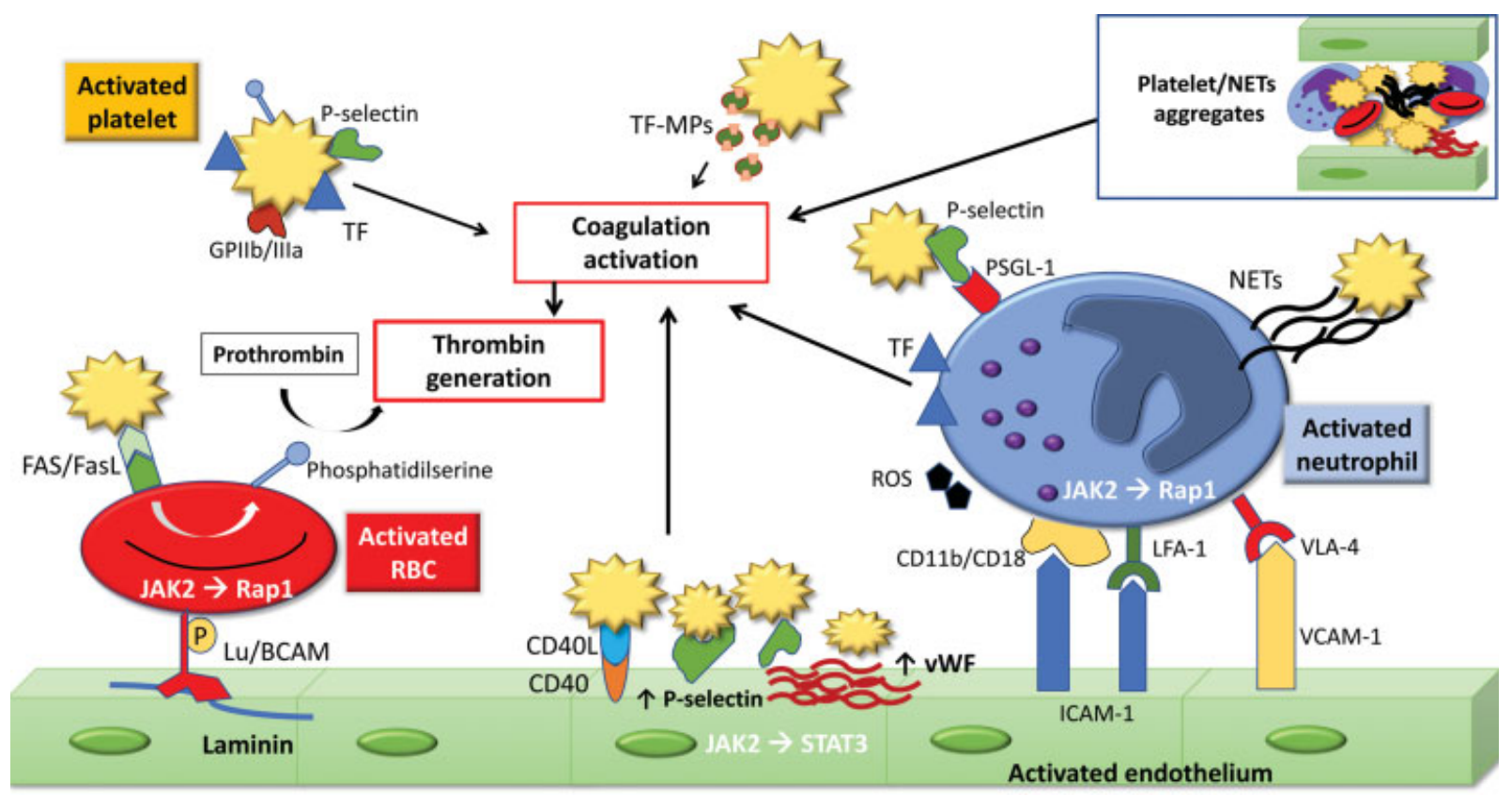

Fig. 1 Pathogenesis of thrombosis in MPN. In RBCs, JAK2V617F mutation mediates the phosphorylation of the erythroid Lutheran/basal celladhesion molecule (Lu/BCAM) through the Rap1/Akt signaling pathway, determining an aberrant adhesion of RBCs to the endothelial cells (EC) ${ }^{92}$ Moreover, RBC/platelet interaction through FAS ligand (FASL)/FAS receptor (FASR) enhances the externalization of RBC phosphatidylserine, favoring thrombin generation and the formation of occlusive thrombi. ${ }^{93}$ Platelets from MPN patients circulate in an activated status, as assessed by the increased expression of procoagulant proteins on their surface such as tissue factor (TF) and P-selectin. ${ }^{12}$ P-selectin is essential for TF accumulation and fibrin formation in the developing thrombus, and also favors platelet/leukocyte interaction via counter-receptor PSGL-1 (P-selectin glycoprotein ligand-1), inducing CD11b and TF upregulation on the neutrophil surface. In turn, CD11b promotes leukocyte adherence to EC and platelets, and release of TF, proteases, and ROS. ${ }^{94}$ MPN leukocytes show increased expression of integrins (i.e., VLA4 and LFA1) that interact with EC extracellular matrix. ${ }^{95}$ Furthermore, neutrophils from JAK2V617F-positive patients are primed to form neutrophil extracellular traps (NETs), ${ }^{96}$ structures composed of DNA, histones, and proteolytic enzymes, implicated in thrombosis as they provide suitable scaffolds for binding RBC, platelets, and von Willebrand factor (VWF). JAK2-mutated endothelium secretes high levels of P-selectin and vWF, and expresses adhesion molecules (i.e., ICAM-1, VCAM-1) and receptors (i.e., CD40), which favor the interaction with platelets and leukocytes, enhancing reciprocal activation. ICAM-1, intracellular adhesion molecule 1; LFA-1, lymphocyte function-associated antigen 1 ; STAT, signal transducer and activator of transcription; VCAM-1, vascular cell adhesion molecule 1; VLA4, very late antigen 4; FasL: Fas ligand. 
intent in selected patients eligible for allogeneic stem cell transplantation.

Thrombosis prevention will be discussed separately for PV, ET, and pre-PMF patients, respectively (-Fig. 2).

\section{Polycythemia Vera}

In low-risk PV patients, aspirin has a confirmed antithrombotic value, ${ }^{46}$ and current recommendations include low-dose aspirin once daily (OD) for all patients. Twice daily (BID) aspirin

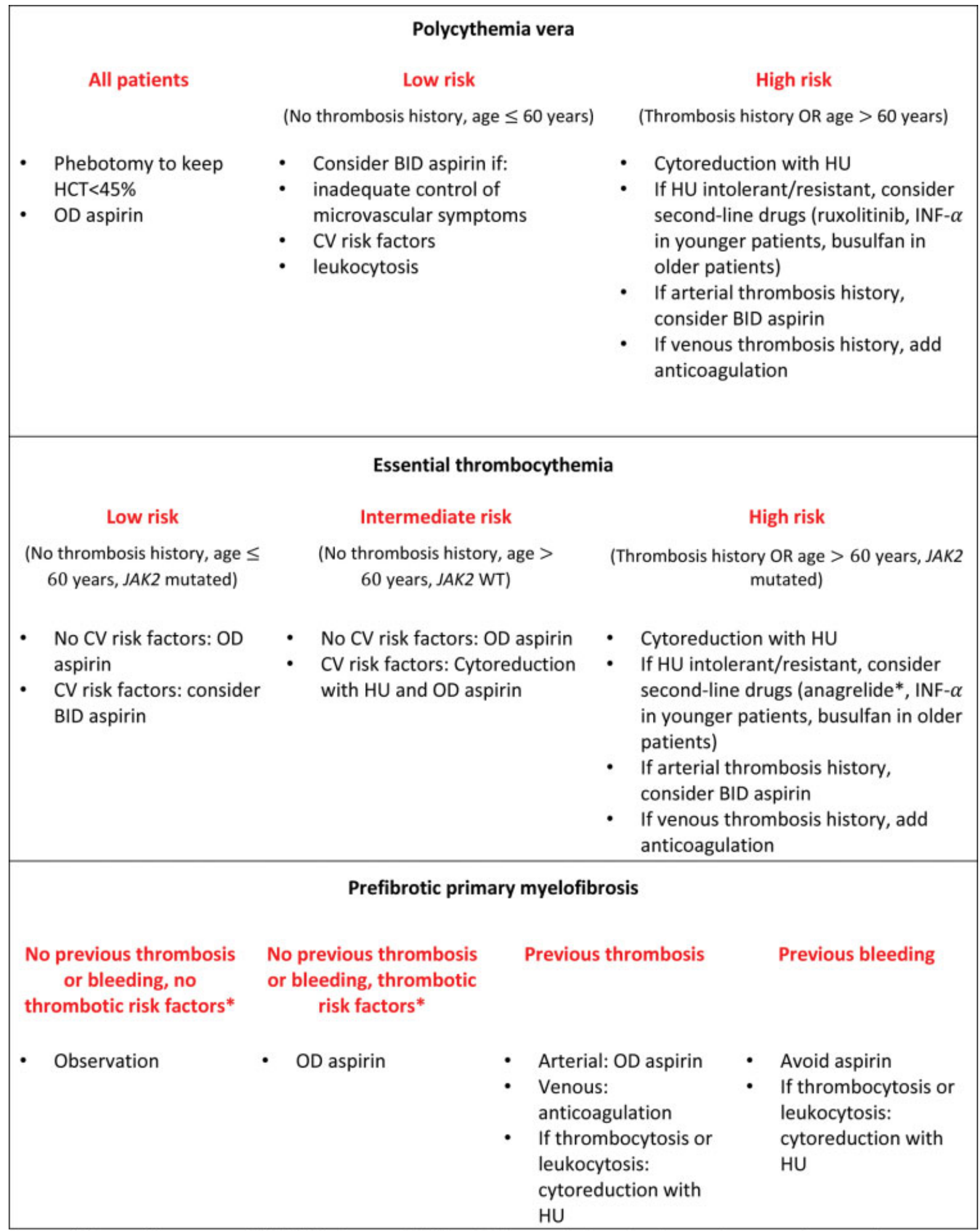

* Age $>60$ years, or CV risk factors, or JAK2 mutation, or leukocytosis, or microvascular symptoms

Fig. 2 Risk-based approach to prophylaxis and treatment of thrombosis in patients polycythemia vera, essential thrombocythemia, and prefibrotic primary myelofibrosis. HCT, hematocrit; OD, once daily; BID, twice daily; CV, cardiovascular; HU, hydroxyurea. 
should be considered in low-risk patients with inadequate control of microvascular symptoms, CV risk factors, or leukocytosis, and in high-risk patients with a history of arterial thrombosis. ${ }^{17}$ Phlebotomy is recommended in all PV patients, and controlled evidence supports the practice of maintaining the hematocrit at less than $45 \%{ }^{29}$ In adjunction, high-risk patients should receive cytoreductive therapy to minimize their risk of thrombosis. Randomized studies in PV have compared hydroxyurea (HU) against pipobroman, the latter being associated with shorter survival, increased risk of leukemic transformation, and a lower risk of post-PV $\mathrm{MF}^{47}$ and radio phosphorus alone or with $\mathrm{HU}$, reporting no difference in survival, incidence of thrombosis, or risk of transformation into post-PV PMF. ${ }^{48}$ Nonrandomized studies have shown a lower incidence of early thrombosis in HU-treated patients compared with historical controls, and an overall low risk of leukemic transformation. ${ }^{49,50}$ To date, $\mathrm{HU}$ is the first-line drug of choice, with a starting dose of $500 \mathrm{mg}$ BID. Two randomized trials (RESPONSE and RESPONSE-2) in PV patients resistant or intolerant to $\mathrm{HU}$, compared the JAK1/2 inhibitor ruxolitinib to best available therapy, including acceptable doses of $\mathrm{HU}$, interferon (INF) or pegylated interferon (pegINF) $\alpha$, pipobroman, anagrelide, lenalidomide/ thalidomide, or no medication, showing a better control in hematocrit levels and symptoms in the ruxolitinib arm. ${ }^{51,52}$ Moreover, a 5-year follow up analysis of the RESPONSE study showed that thrombotic complications were lower in the ruxolitinib group. ${ }^{53}$ Thus, ruxolitinib is approved as secondline therapy in high-risk PV patients. Very recently, ropeginterferon alfa-2b was compared to $\mathrm{HU}$ in a randomized trial (PROUD-PV), and its extension (CONTINUATION-PV). ${ }^{54} \mathrm{Al}-$ though no difference in the rate of complete hematologic response with normal spleen size was observed, ropeginterferon was better in inducing complete hematological response with improved disease burden (i.e. splenomegaly, microvascular disturbances, pruritus, and headache); however, it was associated with a higher liver toxicity, and the follow-up is too short for definitive conclusions. Recent studies have demonstrated a high rate of complete hematologic response, molecular remission, and no risk of leukemic transformation in $\mathrm{HU}$ intolerant/resistant patients treated with busulfan, ${ }^{14,55}$ which is usually reserved to older patients.

\section{Essential Thrombocythemia}

According to the 2021 updated recommendations on PV and ET management, very low-risk patients with ET might not require any therapy unless in the presence of $\mathrm{CV}$ risk factors, where OD low-dose aspirin therapy is advised. ${ }^{17} \mathrm{~A}$ recent study compared the efficacy of OD vs BID and thrice daily (TID) aspirin in ET, based on hypothesis that increased platelet number and turnover might compromise durable inhibition of platelet COX-1; accordingly, BID/TID was more effective than OD dosing in reducing platelet activation, measured by serum thromboxane B2 level. ${ }^{56}$ To date, it seems reasonable to use BID aspirin in patients with arterial thrombosis, or in the presence of $\mathrm{CV}$ risk factors associated to older age or JAK2 mutations. ${ }^{17}$ In the presence of extreme thrombocytosis (platelets $>1,000 \times 10^{9} / \mathrm{L}$ ), the use of aspirin can lead to bleeding complications because of AvWS; in this setting, screening for vWF ristocetin cofactor activity is advised, and aspirin therapy should be withheld with $<20 \%$ vWF activity. Cytoreduction is recommended in patients with intermediate-risk disease and CV risk factors, and in high-risk patients. ${ }^{17} \mathrm{~A}$ controlled study showed superiority of $\mathrm{HU}$ in preventing thrombotic complications in high-risk patients compared to no $\mathrm{HU} .{ }^{31}$ To date, $\mathrm{HU}$ is the first-line drug of choice, and dosing should be titrated to keep platelet count in the normal range, although suggested platelet target is not based on controlled evidence. Two randomized studies compared anagrelide to $\mathrm{HU}$ in $\mathrm{ET}^{7,57}$ In the earlier study, $\mathrm{HU}$ was superior in reducing the risk of arterial thrombosis, major bleeding and fibrotic progression, while anagrelide was more protective in preventing venous thrombosis, although a significantly higher adverse dropout. ${ }^{7}$ In the second study, anagrelide was not inferior to HU in the prevention of thrombotic complications; these results were restricted to patients with ET diagnosed according to the WHO system. ${ }^{57} \mathrm{~A}$ post hoc analysis confirmed a lower rate of venous thrombotic associated to anagrelide, but also a higher rate of hemorrhagic events and arterial thrombosis. ${ }^{58}$ Anagrelide is licensed in some countries (i.e., USA, Japan) as first-line therapy, and in Europe for patients with ET intolerant/refractory to $\mathrm{HU}$, although its use could be proposed in younger patients (i.e. women with child bearing potential) for long term treatment, or as second choice (after INF) in pregnant women. Indeed, INF- $\alpha$ has proved effective in HU intolerant/resistant patients, ${ }^{59}$ and is also associated with significant reduction in mutant CALR allele burden. ${ }^{60}$ Finally, older patients can receive busulfan as second-line treatment. $^{54}$

\section{Prefibrotic Primary Myelofibrosis}

The clinical picture of patients with pre-PMF is heterogeneous, ranging from isolated thrombocytosis, mimicking $\mathrm{ET},{ }^{11}$ to a more aggressive disease. ${ }^{61}$ The risk of vascular events in patients with pre-PMF is similar to that of ET. ${ }^{11,62}$ In studies that evaluated specifically risk factors of thrombosis in prePMF patients evidenced, leukocytosis at diagnosis was a significant risk factor for overall and arterial thrombosis, ${ }^{63,64}$ although leukocyte count during follow-up had no impact in one study ${ }^{64}$ Interestingly, major bleeding seems to occur more frequently in pre-PMF than in ET patients, ${ }^{65}$ where leukocytosis, previous hemorrhage, aspirin therapy, and reticulin grade were found to be predictors of bleeding. ${ }^{65,66}$ In the absence of specific prognostic scores for predicting the risk of bleeding and thrombosis in pre-PMF, a proposed pragmatic approach includes no treatment or low-dose aspirin in asymptomatic patients; aspirin or oral anticoagulation if previous arterial or venous thrombosis, and hydroxyurea as first-line cytoreduction in case of thrombocytosis or leukocytosis. ${ }^{67}$

\section{Management of Arterial Thrombosis}

MPN patients who have a vascular event despite treatment with aspirin require cytoreduction for the management of their blood cell counts. For patients with recurrence of an 
arterial event, aspirin administration may be increased from OD to BID, or clopidogrel may be used instead of aspirin. Correction of $\mathrm{CV}$ risk factors, including blood pressure, lipid levels, smoking, and obesity, is also relevant in MPN patients. In patients at high risk of arterial events, treatment with a broad-spectrum cytoreductive agent, such as HU, rather than a narrow-spectrum strategy, such as anagrelide or phlebotomy, should be considered, to better control all the altered blood counts. ${ }^{68}$

\section{Treatment of Venous Thromboembolism and Secondary Prevention}

Thrombosis recurrences in MPN patients preferentially involve the same arterial or venous districts affected in the first event. ${ }^{69,70}$ Different studies reported as risk factors for recurrence age greater than 60 years and a history of remote thrombosis, ${ }^{69,71}$ but not MPN subtype. ${ }^{69-71}$ After the first episode of VTE, the duration of secondary prophylaxis should be decided balancing the risk of hemorrhagic complications over the benefit of VTE prevention. In the general population, the cumulative rate of recurrence after discontinuation of anticoagulation at 1 and 5 years is 10 and $30 \%$ after unprovoked VTE, respectively, and 5 and 15\% after VTE provoked by nonsurgical reversible factors, respectively. ${ }^{72}$ In MPN patients, the rate of recurrent thrombosis is $6.0,6.5$, and $7.6 \%$ patientyears according to three retrospective studies addressing this issue. $^{69-71}$ Regarding the bleeding risk, results from two of these studies in MPN patients receiving vitamin K antagonist (VKA) treatment are conflicting. ${ }^{70,71}$ In particular, the cumulative probability of major bleeding at 1 year of VKA treatment is $2.8 \%$ in one study, which is higher than the counterpart value of 1.2 to $2.2 \%$ recorded in the VKA arms of trials comparing VKA versus direct oral anticoagulants (DOACs). ${ }^{73-75}$ Moreover, the association of antiplatelet agents plus VKA seems to further increase major bleedings compared with the use of antiplatelet agents or VKA alone. ${ }^{69}$

\section{Acute-Phase Venous Thromboembolism Treatment}

DVT or PE in MPN patients should be approached the same as DVT/PE occurring in the general population. ${ }^{76}$ Therefore, low-molecular-weight heparin (LMWH) or fondaparinux is suggested over intravenous or subcutaneous (SC) unfractionated heparin; early initiation of VKA aiming to target an international normalized ratio of 2.5 (range: 2.0-3.0) is recommended. ${ }^{72}$ Relatively frequent cases of heparin-induced thrombocytopenia (HIT) have been reported in MPN patients, so that special care is due during the heparin course in monitoring a drop of the platelet count. ${ }^{77}$

\section{Long-Term Prophylaxis of Venous Thromboembolism Recurrence}

\section{Vitamin K Antagonists}

In the Italian cohort from the Gruppo Italiano Malattie Ematologiche dell'Adulto(GIMEMA), VKA effectively prevented VTE recurrence in MPN patients. Excluding patients with VTE at unusual sites, long-term treatment with VKA remained effec- tive in preventing recurrence. ${ }^{69}$ In the Spanish cohort from the Grupo Español de Enfermedades Mieloproliferativas Filadelfia Negativas (GEMFIN), VKA treatment was associated with a 2.8 -fold reduction in the risk of thrombotic recurrence. ${ }^{71}$ In the international cohort from the European LeukemiaNet (ELN), the rate of recurrent thrombosis per 100 patient-years was 4.7 on VKA and 8.9 off VKA, and the rate of recurrent VTE was 4.2 among patients who continued VKA and 9.6 after discontinuation of VKA (relative risk: 2.2). After stopping VKA, the recurrence rate at 5 years was $42.3 \%{ }^{70}$ Remarkably, this study evidenced that the cumulative probability of recurrent thrombosis at 1 year of VKA treatment is $7.8 \%{ }^{70}$ particularly high compared with non-MPN population (between 1.8 and 3.5\% according to the most recent studies). ${ }^{73-75}$ Moreover, the cumulative probability of recurrent thrombosis after discontinuation of VKA was $42 \%$ at 5 years, ${ }^{70}$ compared with $29.1 \%$ reported in non-MPN patients. ${ }^{78}$

\section{Low-Molecular-Weight Heparin}

Guidelines for cancer-associated VTE recommend LMWH for at least 3 to 6 months, suggesting to treat indefinitely patients with active malignancy and ongoing anticancer treatment, on the basis of the superior safety and efficacy compared with VKA. ${ }^{79,80}$ However, MPN is a chronic neoplastic disorder; so, continued life-long treatment with daily subcutaneous heparin can be troublesome. Moreover, given the suspected higher risk of HIT in MPN patients, ${ }^{77}$ special caution should be adopted in prescribing long-term treatment. Finally, there is no published evidence about efficacy and safety of long-term administration of LMWH in MPN to establish recommendations for clinical practice.

\section{Cytoreductive Treatment}

Cytoreductive treatment (hydroxyurea in most of the cases) reduced the risk of recurrence of arterial thrombosis in the GIMEMA cohort. ${ }^{69}$ Conversely, in the ELN study, patients receiving VKA without cytoreduction did not show a significant rate of VTE recurrence compared with patients treated with VKA and cytoreduction. ${ }^{70}$ Cytoreductive therapy, along with full anticoagulation, represents the standard of care for MPN patients with splanchnic vein thrombosis (SVT), although a recently proposed treatment algorithm does not recommend cytoreduction in patients with normal blood values. ${ }^{81}$

\section{Direct Oral Anticoagulants}

Dabigatran, rivaroxaban, apixaban, and edoxaban are approved for the treatment of acute VTE. ${ }^{76}$ In the cancer population, three recent randomized clinical trials of DOACs versus LMWH demonstrated the noninferiority of edoxaban, ${ }^{82}$ rivaroxaban, ${ }^{83}$ and apixaban ${ }^{84}$ for VTE treatment in cancer patients. In these trials, the rate of recurrent VTE was lower in the DOACs arm, but in two of them the rate of major bleeding was higher, mainly due to upper gastrointestinal bleeding in patients with gastrointestinal cancer. $^{82,83}$ However, a recent meta-analysis including four randomized controlled studies comparing cancer-associated thrombosis treatment with apixaban, edoxaban, or rivaroxaban with dalteparin showed that the DOACs reduced the risk of 
Table 1 Studies that have evaluated DOACs in MPN patients

\begin{tabular}{|l|l|l|l|l|}
\hline Study & Kaifie et a $^{89}$ & lanotto et al $^{88}$ & Curto-Garcia et al $^{90}$ & Serrao et al $^{91}$ \\
\hline MPN patients & 8 & 25 & 32 & 71 \\
\hline PV & 3 & 8 & 12 & 25 \\
\hline ET & 1 & 17 & 9 & 28 \\
\hline PMF & 4 & 0 & 9 & 13 \\
\hline MPN-U & 0 & 0 & 2 & 5 \\
\hline Type of DOAC & \multicolumn{5}{|l|}{} \\
\hline Rivaroxaban & 8 & 16 & 17 & 26 \\
\hline Apixaban & 0 & 9 & 14 & 21 \\
\hline Edoxaban & 0 & 0 & 1 & 14 \\
\hline Dabigatran & 0 & 0 & 0 & 10 \\
\hline Thrombotic recurrence & - & $1(4)$ & $1(3)$ & 0 \\
\hline Major bleeding & - & $3(12)$ & 0 & 0 \\
\hline CRNMB & - & $2(8)$ & $3(9.3)$ & 0 \\
\hline Median FU (mo) & - & 25 & 25 & 15 \\
\hline
\end{tabular}

Abbreviations: CRNMB, clinically relevant non-major bleeding; DOAC, direct oral anticoagulant; FU, follow-up; MPN-U, myeloproliferative neoplasms unclassifiable.

Source: Data are number (\%).

recurrent VTE with no significant higher likelihood of major bleeding at 6 months compared with $\mathrm{LMWH}^{85}$

Since MPN patients are prone to either thrombotic and hemorrhagic complications, the use of DOACs might reduce the bleeding risk as compared with VKA, ${ }^{86}$ with the capacity to protect from both arterial and venous thrombosis. ${ }^{72}$ However, knowledge of VTE treatment with DOACs in MPN patients is limited. Indeed, in the Hokusai trials, only $10 \%$ of recruited patients had hematological malignancies, leukemia, and lymphoma for most. ${ }^{82,87}$ In the ELN cohort of MPN patients with DVT and/or PE, only $3.3 \%$ of patients were treated with DOACs. ${ }^{70}$ Studies evaluating the use of DOACs in MPN are summarized in - Table 1. In the OBENE registry, of 760 MPN patients, only 13 patients were receiving DOACs for atrial fibrillation (AF) and 8 for VTE. ${ }^{88}$ In the German MPN registry of the Study Alliance Leukemia, 68 of 454 patients (14.9\%) had suffered from DVT or SVT, and only 8 were treated with rivaroxaban. Although nonsignificant, patients on rivaroxaban had a lower incidence of major bleeding as compared with VKA plus double antiplatelet treatment, and to heparin. ${ }^{89}$ Very recently, two studies were published on this issue. ${ }^{90,91}$ A retrospective English study evaluated 32 patients with MPN-associated VTE, including SVT and cerebral thrombosis, receiving DOACs (17 rivaroxaban, 14 apixaban, and 1 edoxaban). ${ }^{90}$ During the follow-up, there were no VTE recurrences in 31 patients, and only one case had evidence of mesenteric ischemia. There were no episodes of major bleeding except for three patients showing clinical relevant non-major (CRNM) bleeding; notably these patients were taking aspirin in addition to a DOAC. A larger Italian study on 71 MPN patients receiving DOAC either for AF or VTE did not record thrombotic complications, nor major or CRNM bleeding after a median follow-up of 12 months. ${ }^{91}$ Notably, 11 patients were treated with ruxolitinib, and no clinical interferences were observed.

\section{Conclusion}

Thrombosis is still a major problem in MPN patients. In the last years, new evidences concerning pathogenesis, risk factors, and treatment options have emerged. Recent reports delineate the increasingly plausible role of various cell adhesion molecules in thrombosis development, which might be explored as possible therapeutic targets. Large prospective studies are needed, especially in low-risk patients, to evaluate the possible advantage of cytoreduction for maintaining WBC within the normal range in reducing thrombotic events. Finally, the therapeutic role of DOACs should be assessed by prospective randomized trials to establish their efficacy and safety, compared with standard treatment, in this particular subset of hematological cancer patients.

Conflict of Interest

The authors declare that they have no conflict of interest.

\section{References}

1 Arber DA, Orazi A, Hasserjian R, et al. The 2016 revision to the World Health Organization classification of myeloid neoplasms and acute leukemia. Blood 2016;127(20):2391-2405

2 Barbui T, Finazzi G, Falanga A. Myeloproliferative neoplasms and thrombosis. Blood 2013;122(13):2176-2184

3 Hultcrantz M, Björkholm M, Dickman PW, et al. Risk for arterial and venous thrombosis in patients with myeloproliferative 
neoplasms: a population-based cohort study. Ann Intern Med 2018;168(05):317-325

4 Rungjirajittranon T, Owattanapanich W, Ungprasert P, Siritanaratkul N, Ruchutrakool T. A systematic review and meta-analysis of the prevalence of thrombosis and bleeding at diagnosis of Philadelphia-negative myeloproliferative neoplasms. BMC Cancer 2019;19(01):184

5 Barbui T, Carobbio A, Rumi E, et al. In contemporary patients with polycythemia vera, rates of thrombosis and risk factors delineate a new clinical epidemiology. Blood 2014;124(19):3021-3023

6 Marchioli R, Finazzi G, Landolfi R, et al. Vascular and neoplastic risk in a large cohort of patients with polycythemia vera. J Clin Oncol 2005;23(10):2224-2232

7 Harrison CN, Campbell PJ, Buck G, et al;United Kingdom Medical Research Council Primary Thrombocythemia 1 Study. Hydroxyurea compared with anagrelide in high-risk essential thrombocythemia. N Engl J Med 2005;353(01):33-45

8 Barbui T, Carobbio A, Cervantes F, et al. Thrombosis in primary myelofibrosis: incidence and risk factors. Blood 2010;115(04): 778-782

9 Carobbio A, Finazzi G, Antonioli E, et al. Thrombocytosis and leukocytosis interaction in vascular complications of essential thrombocythemia. Blood 2008;112(08):3135-3137

10 Passamonti F, Rumi E, Arcaini L, et al. Prognostic factors for thrombosis, myelofibrosis, and leukemia in essential thrombocythemia: a study of 605 patients. Haematologica 2008;93(11): 1645-1651

11 Barbui T, Thiele J, Passamonti F, et al. Survival and disease progression in essential thrombocythemia are significantly influenced by accurate morphologic diagnosis: an international study. J Clin Oncol 2011;29(23):3179-3184

12 Falanga A, Marchetti M. Thrombosis in myeloproliferative neoplasms. Semin Thromb Hemost 2014;40(03):348-358

13 Sekhar M, McVinnie K, Burroughs AK. Splanchnic vein thrombosis in myeloproliferative neoplasms. Br J Haematol 2013;162(06): 730-747

14 Tefferi A, Rumi E, Finazzi G, et al. Survival and prognosis among 1545 patients with contemporary polycythemia vera: an international study. Leukemia 2013;27(09):1874-1881

15 Carobbio A, Thiele J, Passamonti F, et al. Risk factors for arterial and venous thrombosis in WHO-defined essential thrombocythemia: an international study of 891 patients. Blood 2011;117 (22):5857-5859

16 Barbui T, Barosi G, Birgegard G, et al;European LeukemiaNet. Philadelphia-negative classical myeloproliferative neoplasms: critical concepts and management recommendations from European LeukemiaNet. J Clin Oncol 2011;29(06):761-770

17 Tefferi A, Barbui T. Polycythemia vera and essential thrombocythemia: 2021 update on diagnosis, risk-stratification and management. Am J Hematol 2020;95(12):1599-1613

18 Tefferi A, Pardanani A. Myeloproliferative neoplasms: a contemporary review. JAMA Oncol 2015;1(01):97-105

19 Ziakas PD. Effect of JAK2 V617F on thrombotic risk in patients with essential thrombocythemia: measuring the uncertain. Haematologica 2008;93(09):1412-1414

20 Dahabreh IJ, Zoi K, Giannouli S, Zoi C, Loukopoulos D, Voulgarelis M. Is JAK2 V617F mutation more than a diagnostic index? A metaanalysis of clinical outcomes in essential thrombocythemia. Leuk Res 2009;33(01):67-73

21 Klampfl T, Gisslinger H, Harutyunyan AS, et al. Somatic mutations of calreticulin in myeloproliferative neoplasms. N Engl J Med 2013;369(25):2379-2390

22 Rotunno G, Mannarelli C, Guglielmelli P, et al;Associazione Italiana per la Ricerca sul Cancro Gruppo Italiano Malattie Mieloproliferative Investigators. Impact of calreticulin mutations on clinical and hematological phenotype and outcome in essential thrombocythemia. Blood 2014;123(10):1552-1555
23 Rumi E, Pietra D, Ferretti V, et al;Associazione Italiana per la Ricerca sul Cancro Gruppo Italiano Malattie Mieloproliferative Investigators. JAK2 or CALR mutation status defines subtypes of essential thrombocythemia with substantially different clinical course and outcomes. Blood 2014;123(10):1544-1551

24 Finazzi G, Carobbio A, Guglielmelli P, et al. Calreticulin mutation does not modify the IPSET score for predicting the risk of thrombosis among 1150 patients with essential thrombocythemia. Blood 2014;124(16):2611-2612

25 Tefferi A, Barbui T. Essential thrombocythemia and polycythemia vera: focus on clinical practice. Mayo Clin Proc 2015;90(09):1283-1293

26 Pearson TC, Wetherley-Mein G. Vascular occlusive episodes and venous haematocrit in primary proliferative polycythaemia. Lancet 1978;2(8102):1219-1222

27 Di Nisio M, Barbui T, Di Gennaro L, et al;European Collaboration on Low-dose Aspirin in Polycythemia Vera (ECLAP) Investigators. The haematocrit and platelet target in polycythemia vera. Br J Haematol 2007;136(02):249-259

28 Gordeuk VR, Key NS, Prchal JT. Re-evaluation of hematocrit as a determinant of thrombotic risk in erythrocytosis. Haematologica 2019;104(04):653-658

29 Marchioli R, Finazzi G, Specchia G, et al;CYTO-PV Collaborative Group. Cardiovascular events and intensity of treatment in polycythemia vera. N Engl J Med 2013;368(01):22-33

30 Alvarez-Larrán A, Pérez-Encinas M, Ferrer-Marín F, et al;Grupo Español de Neoplasias Mieloproliferativas Filadelfia Negativas. Risk of thrombosis according to need of phlebotomies in patients with polycythemia vera treated with hydroxyurea. Haematologica 2017;102(01):103-109

31 Cortelazzo S, Finazzi G, Ruggeri M, et al. Hydroxyurea for patients with essential thrombocythemia and a high risk of thrombosis. $\mathrm{N}$ Engl J Med 1995;332(17):1132-1136

32 Landolfi R, Cipriani MC, Novarese L. Thrombosis and bleeding in polycythemia vera and essential thrombocythemia: pathogenetic mechanisms and prevention. Best Pract Res Clin Haematol 2006; 19(03):617-633

33 Landolfi R, Di Gennaro L, Barbui T, et al;European Collaboration on Low-Dose Aspirin in Polycythemia Vera (ECLAP) Leukocytosis as a major thrombotic risk factor in patients with polycythemia vera. Blood 2007;109(06):2446-2452

34 Tefferi A. Leukocytosis as a risk factor for thrombosis in myeloproliferative neoplasms-biologically plausible but clinically uncertain. Am J Hematol 2010;85(02):93-94

35 Girodon F, Dutrillaux F, Broséus J, et al. Leukocytosis is associated with poor survival but not with increased risk of thrombosis in essential thrombocythemia: a population-based study of 311 patients. Leukemia 2010;24(04):900-903

36 Cerquozzi S, Barraco D, Lasho T, et al. Risk factors for arterial versus venous thrombosis in polycythemia vera: a single center experience in 587 patients. Blood Cancer J 2017;7(12):662

37 Carobbio A, Ferrari A, Masciulli A, Ghirardi A, Barosi G, Barbui T. Leukocytosis and thrombosis in essential thrombocythemia and polycythemia vera: a systematic review and meta-analysis. Blood Adv 2019;3(11):1729-1737

38 Tefferi A, Barbui T. Polycythemia vera and essential thrombocythemia: 2015 update on diagnosis, risk-stratification and management. Am J Hematol 2015;90(02):162-173

39 Barbui T, Finazzi G, Carobbio A, et al. Development and validation of an International Prognostic Score of thrombosis in World Health Organization-essential thrombocythemia (IPSET-thrombosis). Blood 2012;120(26):5128-5133, quiz 5252

40 Barbui T, Vannucchi AM, Buxhofer-Ausch V, et al. Practice-relevant revision of IPSET-thrombosis based on 1019 patients with WHO-defined essential thrombocythemia. Blood Cancer J 2015; 5:e369

41 Guy A, Gourdou-Latyszenok V, Le Lay N, et al. Vascular endothelial cell expression of JAK2 ${ }^{\mathrm{V} 617 \mathrm{~F}}$ is sufficient to promote a pro- 
thrombotic state due to increased P-selectin expression. Haematologica 2019;104(01):70-81

42 Ataga KI, Kutlar A, Kanter J, et al. Crizanlizumab for the prevention of pain crises in sickle cell disease. N Engl J Med 2017;376(05):429-439

43 Rosti V, Villani L, Riboni R, et al;Associazione Italiana per la Ricerca sul Cancro Gruppo Italiano Malattie Mieloproliferative (AGIMM) investigators. Spleen endothelial cells from patients with myelofibrosis harbor the JAK2V617F mutation. Blood 2013;121(02):360-368

44 Marchetti M, Tartari CJ, Russo L, et al. Phospholipid-dependent procoagulant activity is highly expressed by circulating microparticles in patients with essential thrombocythemia. Am J Hematol 2014;89(01):68-73

45 Marchetti M, Castoldi E, Spronk HM, et al. Thrombin generation and activated protein $C$ resistance in patients with essential thrombocythemia and polycythemia vera. Blood 2008;112(10):4061-4068

46 Landolfi R, Marchioli R, Kutti J, et al;European Collaboration on Low-Dose Aspirin in Polycythemia Vera Investigators. Efficacy and safety of low-dose aspirin in polycythemia vera. $\mathrm{N}$ Engl J Med 2004;350(02):114-124

47 Najean Y, Rain JD. Treatment of polycythemia vera: the use of hydroxyurea and pipobroman in 292 patients under the age of 65 years. Blood 1997;90(09):3370-3377

48 Najean Y, Rain JDThe French Polycythemia Study Group. Treatment of polycythemia vera: use of 32P alone or in combination with maintenance therapy using hydroxyurea in 461 patients greater than 65 years of age. Blood 1997;89(07):2319-2327

49 Fruchtman SM, Mack K, Kaplan ME, Peterson P, Berk PD, Wasserman LR. From efficacy to safety: a Polycythemia Vera Study group report on hydroxyurea in patients with polycythemia vera. Semin Hematol 1997;34(01):17-23

50 Finazzi G, Caruso V, Marchioli R, et al;ECLAP Investigators. Acute leukemia in polycythemia vera: an analysis of 1638 patients enrolled in a prospective observational study. Blood 2005;105 (07):2664-2670

51 Vannucchi AM, Kiladjian JJ, Griesshammer M, et al. Ruxolitinib versus standard therapy for the treatment of polycythemia vera. $\mathrm{N}$ Engl J Med 2015;372(05):426-435

52 Passamonti F, Griesshammer M, Palandri F, et al. Ruxolitinib for the treatment of inadequately controlled polycythaemia vera without splenomegaly (RESPONSE-2): a randomised, open-label, phase 3b study. Lancet Oncol 2017;18(01):88-99

53 Kiladjian J-J, Zachee P, Hino M, et al. Long-term efficacy and safety of ruxolitinib versus best available therapy in polycythaemia vera (RESPONSE): 5-year follow up of a phase 3 study. Lancet Haematol 2020;7(03):e226-e237

54 Gisslinger H, Klade C, Georgiev P, et al;PROUD-PV Study Group. Ropeginterferon alfa-2b versus standard therapy for polycythaemia vera (PROUD-PV and CONTINUATION-PV): a randomised, non-inferiority, phase 3 trial and its extension study. Lancet Haematol 2020;7(03):e196-e208

55 Alvarez-Larrán A, Martínez-Avilés L, Hernández-Boluda JC, et al. Busulfan in patients with polycythemia vera or essential thrombocythemia refractory or intolerant to hydroxyurea. Ann Hematol 2014;93(12):2037-2043

56 Rocca B, Tosetto A, Betti S, et al. A randomized double-blind trial of 3 aspirin regimens to optimize antiplatelet therapy in essential thrombocythemia. Blood 2020;136(02):171-182

57 Gisslinger H, Gotic M, Holowiecki J, et al;ANAHYDRET Study Group. Anagrelide compared with hydroxyurea in WHO-classified essential thrombocythemia: the ANAHYDRET Study, a randomized controlled trial. Blood 2013;121(10):1720-1728

58 Birgegård G, Besses C, Griesshammer M, et al. Treatment of essential thrombocythemia in Europe: a prospective long-term observational study of 3649 high-risk patients in the Evaluation of Anagrelide Efficacy and Long-term Safety study. Haematologica 2018;103(01):51-60
59 Yacoub A, Mascarenhas J, Kosiorek H, et al. Pegylated interferon alfa2a for polycythemia vera or essential thrombocythemia resistant or intolerant to hydroxyurea. Blood 2019;134(18):1498-1509

60 Cassinat B, Verger E, Kiladjian JJ. Interferon alfa therapy in CALRmutated essential thrombocythemia. N Engl J Med 2014;371(02): 188-189

61 Guglielmelli P, Pacilli A, Rotunno G, et al;AGIMM Group. Presentation and outcome of patients with 2016 WHO diagnosis of prefibrotic and overt primary myelofibrosis. Blood 2017;129(24): 3227-3236

62 Rumi E, Boveri E, Bellini M, et al;Associazione Italiana per la Ricerca sul Cancro Gruppo Italiano Malattie Mieloproliferative Investigators. Clinical course and outcome of essential thrombocythemia and prefibrotic myelofibrosis according to the revised WHO 2016 diagnostic criteria. Oncotarget 2017;8(60):101735-101744

63 Buxhofer-Ausch V, Gisslinger H, Thiele J, et al. Leukocytosis as an important risk factor for arterial thrombosis in WHO-defined early/prefibrotic myelofibrosis: an international study of 264 patients. Am J Hematol 2012;87(07):669-672

64 Buxhofer-Ausch V, Gisslinger B, Schalling M, et al. Impact of white blood cell counts at diagnosis and during follow-up in patients with essential thrombocythaemia and prefibrotic primary myelofibrosis. Br J Haematol 2017;179(01):166-169

65 Finazzi G, Carobbio A, Thiele J, et al. Incidence and risk factors for bleeding in 1104 patients with essential thrombocythemia or prefibrotic myelofibrosis diagnosed according to the 2008 WHO criteria. Leukemia 2012;26(04):716-719

66 Campbell PJ, Bareford D, Erber WN, et al. Reticulin accumulation in essential thrombocythemia: prognostic significance and relationship to therapy. J Clin Oncol 2009;27(18):2991-2999

67 Finazzi G, Vannucchi AM, Barbui T. Prefibrotic myelofibrosis: treatment algorithm 2018. Blood Cancer J 2018;8(11):104

68 Sekhar M. Prevention and management of thrombosis in myeloproliferative neoplasms. Clin Adv Hematol Oncol 2017;15(03):178-181

69 De Stefano V, Za T, Rossi E, et al;GIMEMA CMD-Working Party. Recurrent thrombosis in patients with polycythemia vera and essential thrombocythemia: incidence, risk factors, and effect of treatments. Haematologica 2008;93(03):372-380

70 De Stefano V, Ruggeri M, Cervantes F, et al. High rate of recurrent venous thromboembolism in patients with myeloproliferative neoplasms and effect of prophylaxis with vitamin $\mathrm{K}$ antagonists. Leukemia 2016;30(10):2032-2038

71 Hernández-Boluda JC, Arellano-Rodrigo E, Cervantes F, et al; Grupo Español de Enfermedades Mieloproliferativas Filadelfia Negativas (GEMFIN) Oral anticoagulation to prevent thrombosis recurrence in polycythemia vera and essential thrombocythemia. Ann Hematol 2015;94(06):911-918

72 Barbui T, De Stefano V, Falanga A, et al. Addressing and proposing solutions for unmet clinical needs in the management of myeloproliferative neoplasm-associated thrombosis: a consensusbased position paper. Blood Cancer J 2019;9(08):61

73 Bauersachs R, Berkowitz SD, Brenner B, et al;EINSTEIN Investigators. Oral rivaroxaban for symptomatic venous thromboembolism. N Engl J Med 2010;363(26):2499-2510

74 Agnelli G, Buller HR, Cohen A, et al;AMPLIFY Investigators. Oral apixaban for the treatment of acute venous thromboembolism. $\mathrm{N}$ Engl J Med 2013;369(09):799-808

75 Büller HR, Décousus H, Grosso MA, et al;Hokusai-VTE Investigators. Edoxaban versus warfarin for the treatment of symptomatic venous thromboembolism. N Engl J Med 2013;369(15):1406-1415

76 Kearon C, Akl EA, Comerota AJ, et al. Antithrombotic therapy for VTE disease: antithrombotic therapy and prevention of thrombosis, 9th ed: American College of Chest Physicians Evidence-Based Clinical Practice Guidelines. Chest 2012;141(2, Suppl):e419S-e96S

77 Castelli R, Gallipoli P, Schiavon R, Teatini T, Deliliers GL, Bergamaschini L. High prevalence of heparin induced thrombocytopenia with thrombosis among patients with essential 
thrombocytemia carrying V617F mutation. J Thromb Thrombolysis 2018;45(01):106-113

78 Prandoni P, Noventa F, Ghirarduzzi A, et al. The risk of recurrent venous thromboembolism after discontinuing anticoagulation in patients with acute proximal deep vein thrombosis or pulmonary embolism. A prospective cohort study in 1,626 patients. Haematologica 2007;92(02):199-205

79 Lee AY, Levine MN, Baker RI, et al;Randomized Comparison of Low-Molecular-Weight Heparin versus Oral Anticoagulant Therapy for the Prevention of Recurrent Venous Thromboembolism in Patients with Cancer (CLOT) Investigators. Low-molecular-weight heparin versus a coumarin for the prevention of recurrent venous thromboembolism in patients with cancer. $\mathrm{N}$ Engl J Med 2003;349 (02):146-153

80 Lee AYY, Kamphuisen PW, Meyer G, et al;CATCH Investigators. Tinzaparin vs warfarin for treatment of acute venous thromboembolism in patients with active cancer: a randomized clinical trial. JAMA 2015;314(07):677-686

81 Finazzi G, De Stefano V, Barbui T. Splanchnic vein thrombosis in myeloproliferative neoplasms: treatment algorithm 2018. Blood Cancer J 2018;8(07):64

82 Raskob GE, van Es N, Verhamme P, et al;Hokusai VTE Cancer Investigators. Edoxaban for the treatment of cancer-associated venous thromboembolism. N Engl J Med 2018;378(07):615-624

83 Young AM, Marshall A, Thirlwall J, et al. Comparison of an oral factor Xa inhibitor with low molecular weight heparin in patients with cancer with venous thromboembolism: results of a randomized trial (SELECT-D). J Clin Oncol 2018;36(20):2017-2023

84 Agnelli G, Becattini C, Meyer G, et al;Caravaggio Investigators. Apixaban for the treatment of venous thromboembolism associated with cancer. N Engl J Med 2020;382(17):1599-1607

85 Giustozzi M, Agnelli G, Del Toro-Cervera J, et al. Direct oral anticoagulants for the treatment of acute venous thromboembolism associated with cancer: a systematic review and metaanalysis. Thromb Haemost 2020;120(07):1128-1136

86 Gómez-Outes A, Lecumberri R, Suárez-Gea ML, Terleira-Fernández AI, Monreal M, Vargas-Castrillón E. Case fatality rates of recurrent thromboembolism and bleeding in patients receiving direct oral anticoagulants for the initial and extended treatment of venous thromboembolism: a systematic review. J Cardiovasc Pharmacol Ther 2015;20(05):490-500

87 Raskob GE, van Es N, Segers A, et al;Hokusai-VTE Investigators. Edoxaban for venous thromboembolism in patients with cancer: results from a non-inferiority subgroup analysis of the HokusaiVTE randomised, double-blind, double-dummy trial. Lancet Haematol 2016;3(08):e379-e387

88 Ianotto JC, Couturier MA, Galinat H, et al. Administration of direct oral anticoagulants in patients with myeloproliferative neoplasms. Int J Hematol 2017;106(04):517-521

89 Kaifie A, Kirschner M, Wolf D, et al;Study Alliance Leukemia (SAL) Bleeding, thrombosis, and anticoagulation in myeloproliferative neoplasms (MPN): analysis from the German SAL-MPN-registry. J Hematol Oncol 2016;9:18

90 Curto-Garcia N, Doyle AJ, Breen KA, et al. Outcomes of patients receiving direct oral anticoagulants for myeloproliferative neoplasm-associated venous thromboembolism in a large tertiary centre in the UK. Br J Haematol 2020;189(03):e79-e81

91 Serrao A, Breccia M, Napolitano M, et al. A multicenter real-life study on anticoagulant treatment with direct oral anticoagulants in patients with Ph-negative myeloproliferative neoplasms. Am J Hematol 2020;95(12):E329-E332

92 De Grandis M, Cambot M, Wautier MP, et al. JAK2V617F activates Lu/BCAM-mediated red cell adhesion in polycythemia vera through an EpoR-independent Rap1/Akt pathway. Blood 2013;121(04):658-665

93 Klatt C, Krüger I, Zey S, et al. Platelet-RBC interaction mediated by FasL/FasR induces procoagulant activity important for thrombosis. J Clin Invest 2018;128(09):3906-3925

94 Arellano-Rodrigo E, Alvarez-Larrán A, Reverter JC, Villamor N, Colomer D, Cervantes F. Increased platelet and leukocyte activation as contributing mechanisms for thrombosis in essential thrombocythemia and correlation with the JAK2 mutational status. Haematologica 2006;91(02):169-175

95 Edelmann B, Gupta N, Schnoeder TM, et al. JAK2-V617F promotes venous thrombosis through $\beta 1 / \beta 2$ integrin activation. J Clin Invest 2018;128(10):4359-4371

96 Wolach O, Sellar RS, Martinod K, et al. Increased neutrophil extracellular trap formation promotes thrombosis in myeloproliferative neoplasms. Sci Transl Med 2018;10(436):eaan8292 